\title{
Real-time Cartographic Generalisation: Risk Management Maps Case Study
}

\author{
Karel STANEK ${ }^{\mathrm{a}, *}$, Petr SILHAK ${ }^{\mathrm{a}}$ \\ a Masaryk University,Brno, Czech Republic, karst@geogr.muni.cz,petrsilhak@hotmail.com \\ * Corresponding author
}

Keywords: Cartographic generalisation, Thematic maps, Risk management maps, Web cartography

\begin{abstract}
:
One of the main tasks that have been addressed within the project "Dynamic Mapping Methods Focused on Risk and Disaster Management in the Big Data Era" was how to deal with a scale-dependent modification of a thematic content. Thematic content on this type of maps is composed of point-based observations from different sources. Assembled maps are organized according to particular risk phenomena and previously mentioned point observations indicate status of the risk in selected theme. These indicators come from a variety of different sources, such as automatic sensors, volunteer observations, geographically localized patterns on the web and social networks etc. The user of the map has to first select a key indicator and subsequently a set of support indicators, to customize the map to his requirements. Naturally, when scaling down, graphical conflicts between map elements must be resolved. However, the cartographic generalization has to be done in such a way as to also highlight content and preserve the context of presented observations.
\end{abstract}

In the context of the previous paragraph, it was clear that the most suitable cartographic generalization method for our purposes was an aggregation. Generally, the aggregation is a transformation of group map objects into smaller group of map objects (usually on just one target object). In our case we can identify following target objects of the aggregation:

- A multiple observation symbol resulting from aggregation of either several close symbols of the same kind, or several heterogenous symbols to highlight importance or dominance of one specific kind,

- A rectangular array of symbols resulting from aggregation of conglomeration of heterogenous symbols,

- An area that is the aggregation of a bounded array of objects of the same type, areal shape is derived from simplified convex hull of affected symbols, nevertheless, in some cases, the concave hull and best-fit ellipse were also tried,

- An isopleth representation of value regularly distributed symbols of the same kind, where isopleths are derived from IDW interpolation either of the symbol value or by symbol density, border lines of zones are simplified and invisible.

For the purpose of controlling the generalization process, it is necessary to set the conditions for generalization of the map objects. Such conditions - constraints are in this case relatively simple - symbol size is fixed, symbols cannot overlap, symbols are closer to their original location then other symbols (rectangular group of symbols is considered as one aggregated symbol). It is also necessary to choose which transformation mechanism belongs to which group of map objects. Given the small complexity of the situations and the need for rapid identification of the generalization operator, we have chosen rule-based control system. Rule set is extensible and stored in a database, its core is small due to a cover transformation of group of point symbols to one entity expressed by potentially four representations (or its combination). Because isolated objects are rarely present on the map, key role in the generalisation process plays a structure recognition. Process of the structure recognition is triggered by symbol collision. Identified symbol collisions are clustered by distance and clusters are solved together. For each type of symbol present within an individual cluster, cartometric measurement are made in the cluster's vicinity. The resulting parameterization leads to the rule identification. Several possibilities how to prioritize rules, in case of ambiguity and impact of their setting on the generalization process are also further discussed.

Second, but not less important part of the article, is dedicated to the implementation of the cartographic generalisation process. Because the final goal is to build a web map, the implementation is based on web technologies. Core of the implementation is based on node.js server. All processing tools were defined as JavaScript functions. For geoprocessing purposes, open source library Turf was used, because this technology falls under MIT license. Measurements and rules were stored in open source LevelGraph database. This graph database has nowadays no particular advantage in the limited scope of cartographic generalisation process, but it seems to be promising for possible future and more complex projects. Another reason why beforementioned tools were selected is the possibility to transfer the whole generalization process to the client-side web browser. Input thematic data are the result of a query on web service which provide us evaluated 
anomalies and their cross references. For visualisation purposes were used open source interactive web maps library OpenLayers. 